\title{
Alteração de Software de Código Aberto Para Uso no Ensino de Física
}

\author{
Rodrigo Augusto dos Santos Paula ${ }^{1}$, Cleber Ferreira Oliveira ${ }^{2}$, Marco \\ Antônio Manzan Veludo ${ }^{3}$, Paula Teixeira Makamoto ${ }^{4}$ \\ ${ }^{1}$ Mestrando em Educação Tecnológica - Instituto Federal de Educação, \\ Ciência e Tecnologia do Triângulo Mineiro (IFTM) - Uberaba - MG \\ ${ }^{2}$ Mestrando em Educação Tecnológica - Instituto Federal de Educação, \\ Ciência e Tecnologia do Triângulo Mineiro (IFTM) - Uberaba - MG \\ ${ }^{3}$ Mestrando em Educação Tecnológica - Instituto Federal de Educação, \\ Ciência e Tecnologia do Triângulo Mineiro (IFTM) - Uberaba - MG \\ ${ }^{4}$ Orientadora - Instituto Federal de Educação, Ciência e Tecnologia do \\ Triângulo Mineiro (IFTM) - Uberaba - MG \\ \{rodrigo.rodsystem, clebermat2, marcoamv3000\}@gmail.com, \\ paula@iftm.edu.br
}

\begin{abstract}
This article takes its place in the context of the use of a mobile application for learning Physics. The changes made aimed at facilitating the teaching-learning process, for both the teachers and the students using resources where it is possible to see formulas, target audience, use description, access to source code, and translation into Brazilian Portuguese, among other resources..
\end{abstract}

Resumo. Este artigo se insere no contexto do uso de um aplicativo mobile para aprendizado da disciplina de física. As alterações foram com o intuito de facilitar o ensino aprendizagem tanto para os docentes como para dos discentes utilizando recursos onde é possivel ver as fórmulas, público alvo, descrição de utilização, acesso ao código fonte, tradução para o português do Brasil, entre outros recursos.

\section{Introdução}

A escola sempre se notabilizou como único local onde as pessoas aprendem até metade do século XX isso manteve como dogma, no entanto com o avanço das tecnologias móveis e principalmente com o avanço da Computação na Nuvem, esse lugar de conhecimento ganha mais concorrentes e cada vez mais as informações estão disponíveis em diversas formas e lugares, inclusive no próprio corpo das pessoas com os dispositivos Wearables ${ }^{1}$. A Internet das coisas captura dados em tempo real utilizando sensores dos mais diversos tipos, desde uma torradeira até previsão de Tsunamis.

\footnotetext{
${ }^{1}$ Wearable é a palavra que resume o conceito das chamadas "tecnologias vestíveis", que consistem em dispositivos tecnológicos que podem ser utilizados pelos usuários como peças do vestuário. A palavra inglesa wearable significa "vestível” ou "usável”, na tradução literal para a língua portuguesa.
} 
VI Congresso Brasileiro de Informática na Educação (CBIE 2017)

Anais dos Workshops do VI Congresso Brasileiro de Informática na Educação (WCBIE 2017)

De acordo com West e Vooslo (2013), “a educação formal foi confinada às quatro paredes da sala de aula, ao passo que os aparelhos móveis podem transferir a aprendizagem para ambientes que maximizam a compreensão". Assim a proposta deste trabalho é mostrar que a utilização de recursos tecnológicos não apenas para o lazer, pode ser um diferencial único para promover a aprendizagem significativa, tornando o aluno responsável pelo seu conhecimento. O engajamento proposto pelo trabalho está no fato de que os problemas tradicionais estudados em sala de aula podem ser abordados de forma diferente, isto é, utilizar um recurso em que o próprio aluno gere dados do seu deslocamento e os analise acerca da teoria estudada em sala, faz com que os mesmos se sintam motivados a aprender e fazer a suas próprias descobertas.

A partir de um projeto criado e disponibilizado pelo professor Paulo Francisco Slomp da Universidade Federal do Rio Grande do Sul que consistiu em organizar uma lista pública de aplicativos para dispositivos móveis com o Sistema Operacional Android sob a licença General Public Licence $(\mathrm{GPL})^{2}$. Foi realizada uma pesquisa de aplicativos que poderiam ser usados no ensino da disciplina de Física. A escolha foi baseada principalmente na facilidade de uso e se o código fonte estava disponível para acesso e modificação. A figura 1 ilustra a lista usada para encontrar o aplicativo a ser modificado para que possa ser usado no ensino de Física.

\begin{tabular}{|c|c|c|c|c|c|c|c|c|c|c|}
\hline Fisica - Acústica & Não & Não & Não & Não & Sim & 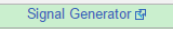 & Android 四 $^{-1}$ & Fonte [ & GNU GPL 四 & $\mathrm{EN}$ \\
\hline Fisica - Astronomia & Não & Não & Não & Sim & Sim & AeonDroid \& & Android $\mathbf{B}^{\circ}$ & Fonte 四 & GNU GPL四 & EN \\
\hline Fisica - Astronomia & Não & Não & Não & Sim & Sim & Antikythera Simulation « & Android G $^{\circ}$ & Fonte [s & Estilo BSD 四 & EN \\
\hline Fisica - Astronomia & Não & Não & Não & Sim & Sim & Constellations [ & Android 四 & Fonte 愙 & MIT X11 G & EN \\
\hline Fisica - Astronomia & Não & Não & Não & Sim & Sim & DroidSat 四 & Android G $^{\circ}$ & Fonte $\mathbb{S}^{\mathrm{S}}$ & GNU GPL 四 & EN \\
\hline Fisica - Astronomia & Não & Não & Não & Não & Sim & Galaxy Zoo & Android s $^{-}$ & Fonte 四 & GNU GPL 四 & EN \\
\hline Física - Astronomia & Não & Não & Não & Sim & Sim & Hubble Gallery [ & Android \& & Fonte [4 & GNU GPL 四 & EN \\
\hline Fisica - Astronomia & Sim & Sim & Não & Não & Não & Moon Phase \& & Android ॠ & Fonte 嵒 & GNU GPL 四 & Sil \\
\hline Fisica - Astronomia & Não & Não & Sim & Sim & Sim & Sky Map 四 & Android 四 & Fonte [s & Apache 2.0 世 & PT \\
\hline Fisica - Atmosfera & Não & Não & Sim & Sim & Sim & AtmosphereLogger [ & Android \& & Fonte 四 & 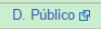 & EN \\
\hline Fisica - Atmosfera & Não & Não & Sim & Sim & Sim & PressureNet t & Android 四 & Fonte [s & GNU GPL 甘 & EN \\
\hline Fisica - Campo magnético & Não & Não & Não & Sim & Sim & Theremin $(\mathrm{Ad})$ स & 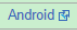 & Fonte [s & GNU GPL 四 & EN \\
\hline Fisica - Conversor de unidades & Não & Não & Sim & Sim & Sim & Units 四 & Android G $^{-}$ & Fonte ब & GNU GPL 因 & EN \\
\hline Fisica - Eletricidade & Não & Não & Sim & Sim & Sim & Ohm Calc 四 & Android s $^{2}$ & Fonte [s & GNU AGPL 四 & EN \\
\hline Fisica - Eletrônica & Não & Não & Não & Sim & Sim & ADSdroid \& & Android $\mathbb{G}^{\circ}$ & Fonte 년 & MIT X11 四 & $\mathrm{EN}$ \\
\hline Fisica - Eletrônica & Não & Não & Não & Sim & Sim & Androsens dassic G & Android 四 & Fonte ब & GNU GPL 㘣 & EN \\
\hline Física - Eletrônica & Não & Não & Não & Sim & Sim & Androsens 2 四 & Android W $^{-}$ & Fonte ब & GNU GPL 圆 & EN \\
\hline Fisica - Eletrônica & Não & Não & Não & Sim & Sim & CurrentWidget $\mathbb{G}^{\prime}$ & Android $\mathbb{G}^{\circ}$ & Fonte $\mathbb{S}^{\mathrm{S}}$ & MIT X11 & $\mathrm{EN}$ \\
\hline Fisica - Eletrônica & Não & Não & Não & Sim & Sim & OsciPrime & Android G $^{-}$ & Fonte ब & GNU GPL \& & EN \\
\hline Fisica - Eletrônica & Não & Não & Não & Não & Sim & RF Analyzer [\$ & Android 四 & Fonte [s & GNU GPL 因 & EN \\
\hline Fisica - Movimento & Não & Não & Não & Sim & Sim & Newton's Cradle US $^{\prime}$ & [Android \&] & Fonte 四 & GNU GPL 田 & $\mathrm{S} / \mathrm{I}$ \\
\hline Fisica - Simulacãao & Não & Não & Não & Sim & Sim & GLXY G & Android \& $^{-1}$ & Fonte 四 & Apache 2.0 世 & EN \\
\hline Fisica - Termodinâmica & Não & Não & Sim & Sim & Sim & Thermometer Extended [ $\sigma^{5}$ & Android 四 & Fonte [s & MIT X11 四 & EN \\
\hline Fisica - Velocidade & Não & Não & Sim & Sim & Sim & Speedmeter 四 & Android W $^{2}$ & Fonte ब & GNU GPL 因 & EN \\
\hline
\end{tabular}

Figura 1. imagem da tabela de aplicativos usados na pesquisa.

\section{Materiais e Métodos}

Devido à grande utilização de Smartphone pelos discentes nas instituições de ensino, acreditamos que será de grande vantagem utilizar estes aparelhos como ferramenta de apoio a aprendizagem, por isso decidimos escolher um software existente na lista da figura 1 para que possamos modificá-lo de acordo com as nossas necessidades e utilizá-lo em sala de aula com os alunos. Dentre os vários softwares existentes na lista, escolhemos um que se chama Speedmeter que pode ser utilizado em

\footnotetext{
${ }^{2}$ A GPL é a licença com maior utilização por parte de projetos de software livre, em grande parte devido à sua adoção para o projeto GNU e o sistema operacional GNU/Linux.
} 
VI Congresso Brasileiro de Informática na Educação (CBIE 2017)

Anais dos Workshops do VI Congresso Brasileiro de Informática na Educação (WCBIE 2017)

aulas de Física para o estudo de velocidade e pode ser instalado em aparelhos que utilizam o sistema operacional Android ${ }^{3}$.

O software Speedmeter conforme a figura 2 foi desenvolvida utilizando a linguagem de programação JAVA, à interface de uso do aplicativo escolhido é composta de informações na língua inglesa e tem como pré-requisito a necessidade de GPS $^{4}$ embutido no aparelho que for executar esta aplicação.

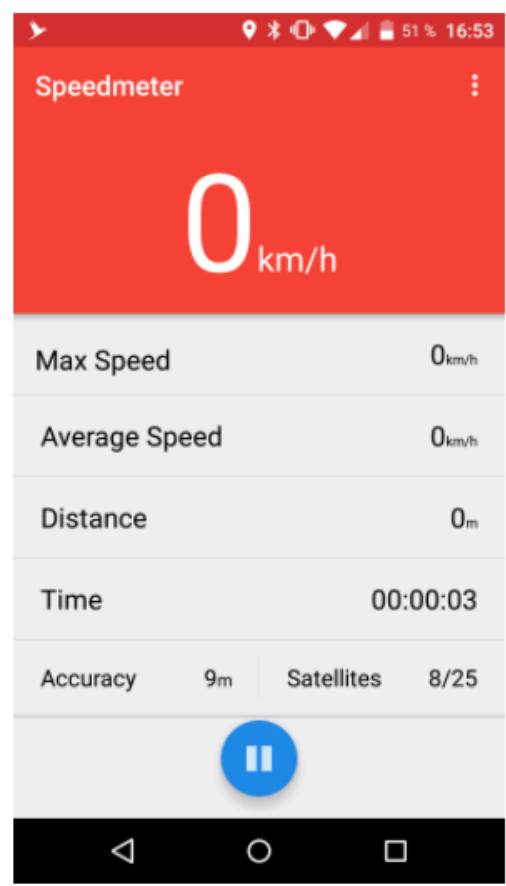

Figura 2. Interface de utilização do software Speedmeter.

Para simplificar o uso do aplicativo pelos discentes, optamos por alterar o código fonte e traduzir as palavras do inglês para o português além de incluir novos recursos como a demonstração das fórmulas de física que são utilizadas. Conforme figura 3 é apresentado a interface do EduSpeedMeter com as alterações implementadas.

\footnotetext{
3 Android é um sistema operacional (SO) baseado no núcleo Linux e atualmente desenvolvido pela empresa de tecnologia Google. Projetado principalmente para dispositivos móveis com tela sensível ao toque como smartphones e tablets.

${ }^{4}$ GPS é a sigla para Global Positioning System, que em português significa "Sistema de Posicionamento Global", e consiste em uma tecnologia de localização por satélite.
} 
VI Congresso Brasileiro de Informática na Educação (CBIE 2017)

Anais dos Workshops do VI Congresso Brasileiro de Informática na Educação (WCBIE 2017)

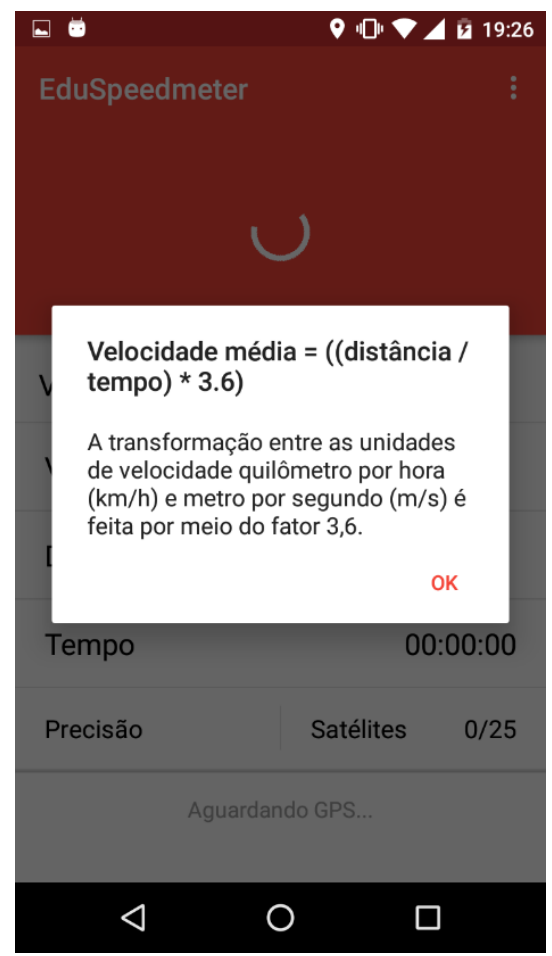

Figura 3: Interface de utilização do software Speedmeter modificada.

Acreditamos que através do uso deste aplicativo com as respectivas alterações, o professor de Física ou de outras disciplinas, poderá proporcionar uma aula diversificada para seus alunos, modificando o formato da aula tradicional e utilizando um recurso que o aluno já está integrado, despertando assim um interesse maior dos mesmos. Como exemplo prático de uso, podemos recomendar que o professor saia da sala de aula juntamente com os alunos em direção a quadra de esportes. Chegando lá os alunos com os seus próprios smartphones com o aplicativo Speedmeter instalado já com as nossas modificações, poderão participar de competições, como uma corrida, por exemplo, onde o aplicativo irá demonstrar a velocidade máxima, velocidade média, distância percorrida, tempo de uso após a confirmação de início do deslocamento e a precisão de cada aluno que participou da brincadeira.

\section{Considerações Finais}

O aplicativo Speedmeter foi uma ótima criação, e graças ao bom senso de seu criador, Ronan Timinello, um estudante da Faculdade de Ciências de Montpellier na França em disponibilizar o código fonte, foi possível realizar algumas melhorias de modo que fosse adaptado às nossas necessidades. Não podemos deixar de citar também a ótima iniciativa do professor Paulo Francisco Slomp e pelo estudante André Ferreira Machado, ambos da UFRGS (Universidade Federal do Rio Grande do Sul) de criarem uma tabela que reúne 305 aplicativos, que podem ser usados como complemento para o processo de ensino-aprendizagem de diversas disciplinas, onde os mesmos tiveram o cuidado de separar os aplicativos por Nível de Ensino, sendo educação infantil, anos iniciais do ensino fundamental, ensino médio e ensino superior. Além de separar as áreas de conhecimento, nome do aplicativo e pagina oficial, link para baixar o aplicativo para funcionamento no sistema operacional Android e código fonte, demonstração do tipo de licença de cada software e o idioma de cada um. 
VI Congresso Brasileiro de Informática na Educação (CBIE 2017)

Anais dos Workshops do VI Congresso Brasileiro de Informática na Educação (WCBIE 2017)

A tabela disponibilizada além de possuir a versão em português, também possui a versão em inglês, espanhol, francesa e italiana. Speedmeter foi apenas um aplicativo que escolhemos entre vários existentes nesta tabela e acreditamos que assim todos podem criar um ciclo de aprendizagem compartilhada e de inteligência coletiva.

\section{Referências}

West, Mark, Vooslo, Seteven, "Policy Guidelines for Mobile Learning (2013)", http://unesdoc.unesco.org/images/0022/002277/227770por.pdf, Acesso em $18 / 08 / 2017$

Brito, Edivaldo. Saiba o que é GNU e qual seu papel no movimento do software livre. 2012. Disponível em: <http://www.techtudo.com.br/artigos/noticia/2012/12/saiba-oque-e-gnu-e-qual-seu-papel-no-movimento-do-software-livre.html $>$. Acesso em: $19 / 08 / 2017$.

Universidade Federal do Rio Grande do Sul. (29 de 06 de 2017). Software Educacional Livre para Dispositivos Móveis - Tabela Dinâmica - Wiki Software Livre na Educação. Fonte: https://www.ufrgs.br/soft-livreedu/wiki/Software_Educacional_Livre_para_Dispositivos_M\%C3\%B3veis__Tabela_Din\%C3\%A2mica. Acesso em 14/09/2017 